\title{
miR-96-5p Suppresses the Progression of Nasopharyngeal Carcinoma by Targeting CDKI
}

This article was published in the following Dove Press journal: OncoTargets and Therapy

\author{
Xiaoqin Luo (D) \\ Xian $\mathrm{He}^{\prime}$ \\ Xing Liu $^{2}$ \\ Lunkun Zhong' \\ Wenjian $\mathrm{Hu}^{\prime}$ \\ 'Department of Otolaryngology, \\ Affiliated Traditional Chinese Medicine \\ Hospital of Southwest Medical University, \\ Luzhou, Sichuan 646699, People's \\ Republic of China; ${ }^{2}$ Department of \\ Urology, Affiliated Traditional Chinese \\ Medicine Hospital of Southwest Medical \\ University, Luzhou, Sichuan 646699 \\ People's Republic of China
}

Background: Nasopharyngeal carcinoma (NPC) is a malignant tumor that occurs in the nasopharyngeal mucosa. Clinically, radiotherapy is the preferred treatment for NPC, and cervical lymph node metastasis is easy to emerge in the early stage. Therefore, this study aimed to investigate the role and potential molecular mechanisms of miR-96-5p in NPC cells to develop new therapeutic horizons.

Methods: The expression of miR-96-5p and CDK1 was measured by RT-qPCR or Western blot. The target relationship between miR-96-5p and CDK1 was confirmed by luciferase reporter assay. CCK-8, sphere formation, flow cytometry and colony formation assay were employed to examine cell viability, stem-like property, apoptosis and cycle, respectively. Male BALB/c nude mice model (6-8 weeks, weigh 18-20 g) was used to evaluate the effect of miR-96-5p on tumor growth in vivo.

Results: miR-96-5p was lowly expressed and CDK1 was highly expressed in NPC tissues and cell lines. CDK1 was identified as a direct target of miR-96-5p, and its expression was negatively regulated by miR-96-5p. By targeting CDK1, miR-96-5p overexpression significantly inhibited tumor sphere formation, promoted apoptosis and cell cycle arrest in CNE-2Z cells. Importantly, CCK-8 and colony formation assay demonstrated that elevated miR-96-5p enhanced the radiotherapy and chemotherapy sensitivity of CNE-2Z cells. Animal experiments showed that the overexpression of miR-96-5p reduced tumor weight and size in tumorbearing mice and inhibited the expression of stem-like marker proteins and apoptosis-related proteins.

Conclusion: These results, together, suggested that miR-96-5p induced cell cycle arrest and apoptosis, inhibited stem-like property, and enhanced the radiochemical sensitivity of NPC by targeting CDK1. In short, miR-96-5p may be a diagnostic and therapeutic target for NPC. Keywords: nasopharyngeal carcinoma, miR-96-5p, CDK1, stem-like property, radiochemosensitivity

\section{Introduction}

Nasopharyngeal carcinoma (NPC) is one of the most common squamous cell carcinomas involving the nasopharyngeal epithelium. ${ }^{1}$ In China, the incidence rate of NPS is nearly 20 times that of in Western countries. ${ }^{2}$ Currently, no significant improvement in overall survival ${ }^{3}$ of NPC patients has been observed even with radiochemotherapy for NPC. ${ }^{4}$ Since the pathogenesis of NPC has not been fully elucidated, it is urgent to seek effective treatment strategies.

It is well known that microRNAs (miRNAs) are a small class of noncoding single stranded RNA, which act a pro-cancer or anti-cancer role in the malignant progression of different tumors, such as colorectal cancer, ${ }^{5}$ oral cancer, ${ }^{6}$ lung
Correspondence: Wenjian $\mathrm{Hu}$ Department of Otolaryngology, Affiliated Traditional Chinese Medicine Hospital of Southwest Medical University, Luzhou City, Sichuan Province 646699,

People's Republic of China

Tel +86 I3980247707

Email bIr4n0acptk4@sina.com 
cancer $^{7}$ and NPC. ${ }^{1}$ MiR-96-5p, a member of the miR-18396-182 cluster, plays an important role in tumor regulation. ${ }^{8}$ Importantly, miR-96-5p has been studied as an oncogene and bio-marker of oral tumorigenesis. ${ }^{9}$ Furthermore, miR-96-5p has carcinogenic effects in the progression of colorectal cancer. ${ }^{10}$ However, the role and mechanism of miR-96-5p in nasopharyngeal carcinoma remains unknown.

Cyclin-dependent kinase 1 (CDK1) is a cell cycle regulator that interacts with cyclin $\mathrm{B}$ to drive the $\mathrm{G} 2-\mathrm{M}$ transition and binds to other interphase cyclin (cyclin D1, $\mathrm{E}$ and $\mathrm{A})$ to regulate $\mathrm{G} 1$ progression and G1-S transition. ${ }^{11,12} \mathrm{CDK} 1$ may regulate apoptosis through caspase phosphorylation. ${ }^{13,14}$ In addition, studies have demonstrated that CDK1 plays an important role in maintaining pluripotency and genomic stability of human pluripotent stem cells. ${ }^{15}$ CDK1 is overexpressed in cancers and inhibition of CDK1 is considered a potential cancer treatment. $^{16}$

In this study, we detected the miR-96-5p expression in NPC tissues and cells. Also, we investigated its effect on the cell viability, cell cycle, apoptosis, stem-like property and radio-chemosensitivity of NPC cells as well as the interaction between miR-96-5p and CDK1, to provide a basis for the study of NPC targeted therapy.

\section{Materials and Methods}

\section{Tissue Samples}

A total of 49 NPC tissues and para-carcinoma normal tissues (5 $\mathrm{cm}$ away from tumors) were obtained from affiliated traditional Chinese medicine hospital of southwest medical university, and immediately collected in liquid nitrogen. Before obtaining these specimens of fresh tissues, all NPC patients signed a written informed consent. This standard was strictly in accordance with the Declaration of Helsinki (2013 revision) and approved by the ethics committee of southwest medical university.

\section{Cell Culture}

Human immortalized nasopharyngeal epithelial cell line NP69 and NPC cell lines (CNE-2Z, HNE-2, C666-1, 5$8 \mathrm{~F}$ and SUNE-1) were obtained from China Center for Type Culture Collection (Wuhan, China). These six cells were kept in RPMI-1640 or DMEM (High Glucose) medium (Thermo Fisher, Waltham, USA) supplemented with $10 \%$ fetal bovine serum (FBS, Gibco, CA, USA), penicillin $(100 \mathrm{U} / \mathrm{mL})$, and streptomycin $(100 \mathrm{mg} / \mathrm{mL})$, and were grown in a $5 \% \mathrm{CO}_{2}$ incubator at $37^{\circ} \mathrm{C}$. Cisplatin (DDP)resistant (CNE-2Z-DDP) and radiotherapy-resistant (CNE2Z-R) cell lines established as previously described. ${ }^{17,18}$

\section{Transfection}

Briefly, CNE-2Z cells were plated and grown to $80-90 \%$ confluence, each well was added with $2 \mathrm{~mL}$ medium without antibiotics. CDK1 overexpression vector (pc-CDK1), miR96-5p mimics or $\mathrm{NC}$ mimics were synthesized by GenePharma (Shanghai, China). According to the manufacturer's instructions, CNE-2Z cells were transfected using Lipofectamine 2000 (Invitrogen, CA, USA) and were harvested $24 \mathrm{~h}$ after transfection for the following experiments.

\section{Real-Time Quantitative Polymerase Chain Reaction (RT-qPCR)}

RNA was extracted with TRIzol reagent (Invitrogen, CA, USA), according to the manufacturer's instructions. RTqPCR was performed to determine the expression of miR96-5p or CDK1 using SYBR mix kit (Takara, Otsu, Japan) on iQ5 RealTime PCR System (BioRad, California, USA). Data were normalized with the $2^{-\Delta \Delta \mathrm{Ct}}$ means, and the primers were listed below:

miR-96-5p-F: 5'-ATGCTTTCTCAACTTGTTGG-3', miR-96-5p-R: 5'-TCACCG CTCTTGGCCGTCACA-3'; CDK1-F: 5'-CAATGACCCCGCACGATTTC-3', CDK1-R: 5'-CATGGAGGGCGGATTGGAA-3'; GAPDH-F: 5'-AACGGATTTGGTCGTATTGGG-3', GAPDH-R: 5'-TCGCTCCTGGAAGATGGTGAT-3'; U6-F: 5'-AGGGGCCATCCACAGTCTTC-3', U6-R: 5'-AACGCTTCACGAATTTGCGT-3'.

\section{Sphere Formation Assay}

CNE-2Z cells with a concentration of about $1.5 \times 10^{3}$ cells/ $\mathrm{mL}$ were seeded in 6-well plates (Corning, NY, USA) and cultured in serum-free DMEM-F12 medium (Gibco, CA, USA) supplemented with $20 \mathrm{ng} / \mathrm{mL}$ EGF (Sigma-Aldrich, St. Louis, USA), 20ng/mL bFGF (Sigma-Aldrich, St. Louis, USA) and 1\% B27 (Invitrogen, CA, USA). Post 14 days, the number of spheres was captured and quantified using an inverted microscope (Zeiss, Oberkochen, Germany). ${ }^{19}$

\section{Radio-Chemosensitivity Administration}

CNE-2Z, CNE-2Z-DDP and CNE-2Z-R cells were seeded into a 96 -well plate $\left(5 \times 10^{3}\right.$ cells/well) and incubated for 24 h. CNE-2Z and CNE-2Z-DDP were treated with DDP 
of $0,2.5,5.0,10$ or $20 \mu \mathrm{M}, \mathrm{CNE}-2 \mathrm{Z}$ and CNE-2Z-R were irradiated with X-rays of $0,2,4,6$ or $8 \mathrm{~Gy}$. Then, these cells were employed for subsequent clone formation and CCK-8 assay.

\section{Clone Formation Assay}

Post transfection $24 \mathrm{~h}$, the $1 \times 10^{5}$ CNE-2Z, CNE-2Z-DDP and CNE-2Z-R cells were uniformly planted in a 6-well plate (Corning, NY, USA) and incubated in a $5 \% \mathrm{CO}^{2}, 37^{\circ}$

$\mathrm{C}$ environment for 2 weeks. After that, the cell colonies were fixed with $10 \%$ formaldehyde (Beyotime, Haimen, China) and stained with $1 \%$ crystal violet (Beyotime, Haimen, China) for $5 \mathrm{~min}$. The visible colonies were captured under an inverted microscope (Zeiss, Oberkochen, Germany) and counted by a Digital Imaging Software QCapture Pro 7 (EBSCO Industries, Inc., Birmingham, USA).

\section{Cell Viability (CCK-8) Assays}

$1 \times 10^{3}$ transfected cells (CNE-2Z, CNE-2Z-DDP and CNE-2Z-R) were cultured in a 96-well plate for $24 \mathrm{~h}$, respectively. Post radio-chemosensitivity administration, cells were supplemented with $10 \mu \mathrm{L}$ CCK-8 solution (Sigma-Aldrich, St. Louis, USA) for another 2 $\mathrm{h}$ incubation, according to the manufacturer's instructions. Finally, the absorbance at $450 \mathrm{~nm}$ was measured using a microplate reader (Bio-Rad, California, USA). Cell survival rate $=(\mathrm{OD}$ value in treated group-OD value in control group)/(OD value in the non-treated group-OD value in control group) $\times 100 \%$.

\section{Luciferase Activity Assay}

The interaction relationship between has-miR-96-5p and CDK1 3'UTR was predicted by targetscan software (http:// www.targetscan.org/vert 72/). CDK1 3' UTR wild-type (wt) or mutant (mut) sequences targeting miR-96-5p were constructed by GenScript (Nanjing, China). The cells were transfected with miR-96-5p mimic and CDK1 3' UTR (wt) or CDK1 3' UTR (mut) using Lipofectamine 2000 (Invitrogen, CA, USA). Luciferase activity was evaluated at $48 \mathrm{~h}$ by Dual-Luciferase Reporter Assay System (Promega, WI, USA).

\section{Western Blot (WB)}

Whole cell lysates were prepared in RIPA buffer (Beyotime, Haimen, China). Proteins were separated by SDS-PAGE, followed by protein concentration measurement, and then were transferred onto PVDF membrane.
After the membranes were blocked with 5\% skim milk, they were incubated with primary antibodies against CDK1 (ab133327, 1:10,000), Bmi1 (ab126783, 1:10,000), SOX2 (ab93689, 1:100), NANOG (ab109250, 1:1000), ALDH1A1 (ab215996, 1:1000), Bax (ab32503, 1:1000), Bcl-2 (ab32124, 1:1000), cleaved Caspase-3 (ab2302, 1:1000), cleaved Caspase-9 (ab32539, 1:1000), cyclinB1 (ab32053, 1:5000) and Human 14-3-3- $\sigma$ antibody (AF44241, $1 \mu \mathrm{g} / \mathrm{mL}$ ) overnight at $4^{\circ} \mathrm{C}$. The next day, the samples were supplemented with the corresponding secondary antibody at room temperature for $1 \mathrm{~h}$. Protein bands were visualized using ECL substrates (BioRad, \#1705061) and imaged on Kodak X-OMAT 2000A (Rochester, NY, USA). Human14-3-3- $\sigma$ antibody was purchased from R\&D Systems (Minnesota, USA), and the rest from Abcam China (Shanghai, China).

\section{Animal Model in vivo}

All animal experiments were conducted in accordance with NIH guidelines for the care and use of experimental animals and approved by the southwest medical university. CNE-2Z cells stably transfected with miR-96-5p mimics or $\mathrm{NC}$ were injected subcutaneously into the right side of male BALB/C nude mice (6-8 weeks, 18-20 g) from Beijing Vital River Laboratory Animal Technology Company (Beijing, China) to establish a xenograft tumor model, tumor weight or volume of mice were checked every 3 day after 7 days. The mice were randomly divided into two groups $(n=10)$ : the NC group and the miR-96-5p mimic group. Twenty-five days later, mice were sacrificed, and the tumor tissues were collected for RT-qPCR, WB or IHC analysis, volume $=\left(\right.$ length $\times$ width $\left.{ }^{2}\right) / 2$.

\section{Immunohistochemistry Assay (IHC)}

Post $10 \%$ formalin fixation, the tumor tissues were dewaxed and rehydrated. The sections were then blocked for $1 \mathrm{~h}$ in PBS with 10\% normal goat serum. After that, the sections were incubated with ALDH1A1 or cleaved Caspase-3 antibody (Abcam China, Shanghai, China). Next day, the sections were rinsed and incubated with HRP-labeled secondary antibodies for $30 \mathrm{~min}$ at $37^{\circ} \mathrm{C}$, and the visual images were captured using an optical microscopy (Leica, Wetzlar, Germany).

\section{Statistical Analysis}

The results are presented as the mean \pm the standard deviation (S.D.), and was repeated in replicate. SPSS 22.0 (IBM, Chicago, USA) was used for statistical 
analysis, one-way ANOVA was employed to analyze the data differences. The difference was considered statistically significant at $P<0.05$.

\section{Results}

\section{miR-96-5p Was Lowly Expressed and CDKI Was Highly Expressed in NPC}

\section{Tissues and Cells}

As shown in Figure 1A and B, the expression of miR96-5p in tumor tissues was significantly lower than that in adjacent normal tissues. On the contrary, the mRNA level of CDK1 in tumor tissues was significantly higher than that in adjacent normal tissues. In addition, the mRNA levels of miR-96-5p and CDK1 in NPC cell lines (CNE-2Z, HNE-2, C666-1, 5-8F and SUNE 1) and normal nasopharyngeal epithelial cell line NP69 were detected by RT-qPCR. Results in Figure 1A and $B$ revealed that the expressions of CDK1 in NPC cell lines were up-regulated, while the expressions of miR96-5p in NPC cell lines were down-regulated, which were consistent with those in tissues. Notably, the differential expression of miR-96-5p or CDK1 was most significant in CNE-2Z cells (Figure 1A and B). Also, Western blot assay showed that CDK1 protein expression in tumor tissues was higher than that in adjacent normal tissues (Figure 1C). Hence, we chose CNE-2Z cells for further study.

\section{CDKI Was a Target of miR-96-5p}

Targetscan predicted that CDK1-3'-UTR was targeted by miR-96-5p between site 585 and 591 (Figure 2A). Then, the dual luciferase reporter assay was used to assess whether CDK1 was a direct target for miR-96-5p. As shown in Figure 2B, wt-CDK1-3'-UTR luciferase activity was inhibited by miR-96-5p mimic, while mut-CDK1-3'UTR luciferase activity did not change significantly. Furthermore, we detected the mRNA levels of miR-96$5 p$ and CDK1 by RT-qPCR. Figure $2 \mathrm{C}$ shows a significant increase in mRNA levels of miR-96-5p, indicating its successful transfection. The relative mRNA level of CDK1 in miR-96-5p mimic cells was significantly lower than that in control groups (Figure 2D).

\section{miR-96-5p Inhibited the Stem-Like Properties of NPC Cells}

To assess the effect of miR-96-5p and on NPC stem cells, stem-like properties were detected by spheroid colony formation assay. As shown in Figure 3A, miR96-5p overexpression significantly reduced the pelletforming ability of cells, while CDK1 overexpression showed the opposite effect. In addition, Western blot further demonstrated that miR-96-5p overexpression increased the expression of stem-like properties marker proteins (Bmi-1, SOX2, NANOG and ALDH1A1) in cells, while CDK1 decreased their expression, and miR-96-5p weakened the downregulation effect of CDK1 on these proteins after miR-96-5p and CDK1 co-transfection (Figure 3B and C).

\section{miR-96-5p Promoted the Apoptosis and Cell Cycle Arrest of NPC Cells}

As shown in Figure 4A and B, flow cytometry showed that miR-96-5p overexpression significantly promoted CNE-2Z apoptosis while CDK1 overexpression inhibited its apoptosis, compared with control. In addition, Western blot further demonstrated that miR-96-5p overexpression increased the expression of Bax, cleaved Caspase- 3 and cleaved Caspase-9 in cells and decreased Bcl-2 expression. CDK1 inversely altered these proteins content (Figure 4C and D). Furthermore, miR-96-5p overexpression promoted cell cycle arrest in $\mathrm{G} 2 / \mathrm{M}$ phase (Figure 4E), and inhibited the expression of cellcycle-associated proteins (14-3-3- $\sigma$ and cyclinB1) (Figure 4F and G). After co-transfection of miR-96-5p mimic and pc-CDK1, miR-96-5p reversed the inhibitory effect of CDK1 on apoptosis and cell cycle of CNE-2Z cells.

\section{miR-96-5p Increased the Chemoradiotherapy Sensitivity of CNE-2Z Cells}

To investigate the role of miR-96-5p in chemoradiotherapy resistance in NPC, we overexpressed miR96-5p in CNE-2Z-R or CNE-2Z-DDP cells and treated with different doses of radiation or DDP, respectively. As shown in Figure 5A, under 0, 2, 4, 6 or 8 Gy X-ray irradiation, cell colonies formed by CNE-2Z-R decreased with dose gradient irradiation, and the colony number of miR-96-5p mimic was higher than that in NC group. The cell survival curve showed that the cell survival fraction (SF) decreased with the ascended of the ascended irradiation. Compared with the NC, the survival curve in mimic group shifted to the right. Notably, the reduction of cell SF in CDK1 group was 
A

miR-96-5p expression in tissues and cells
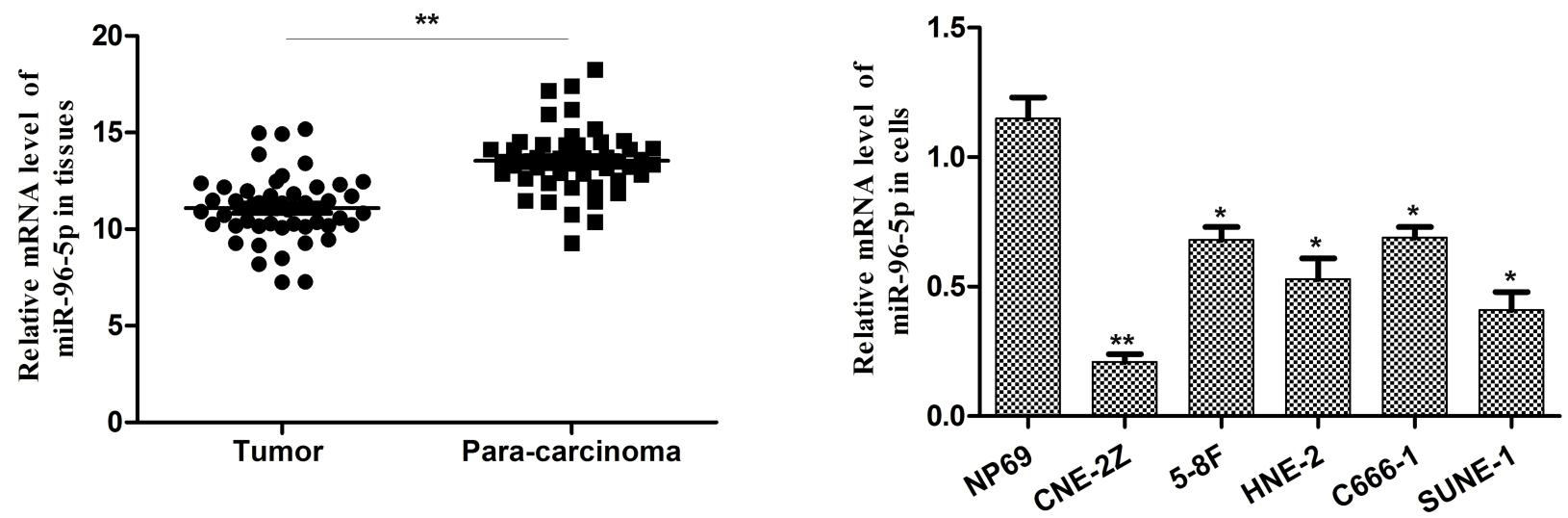

B

CDK1 expression in tissues and cells
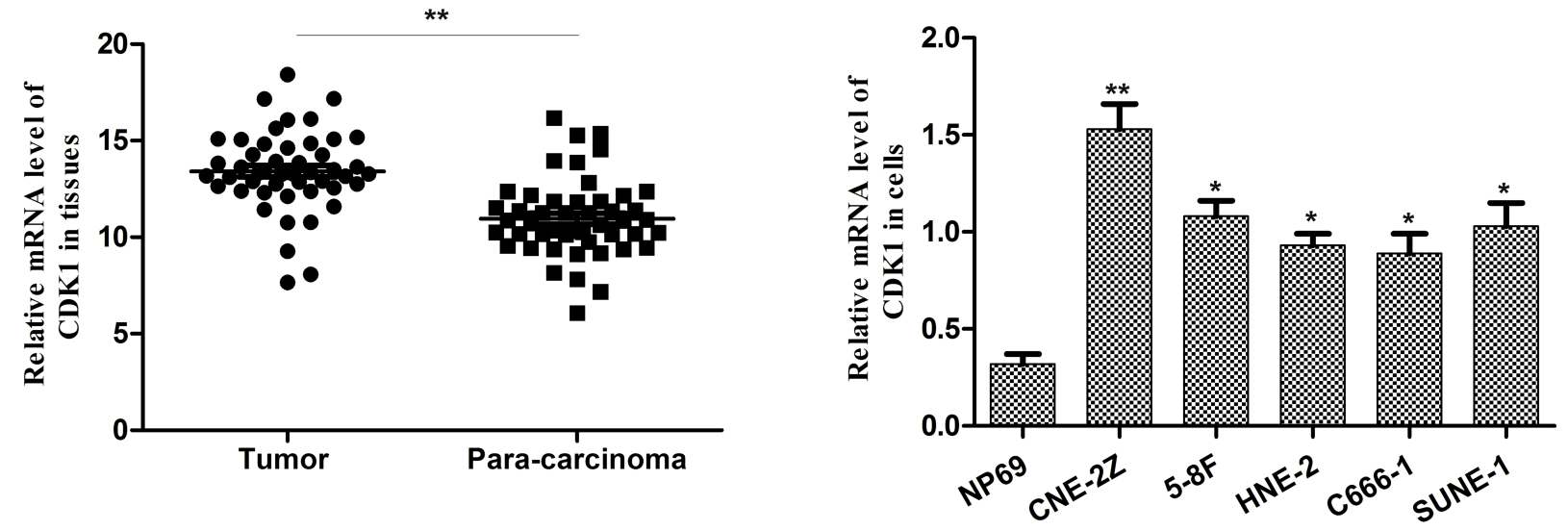

C
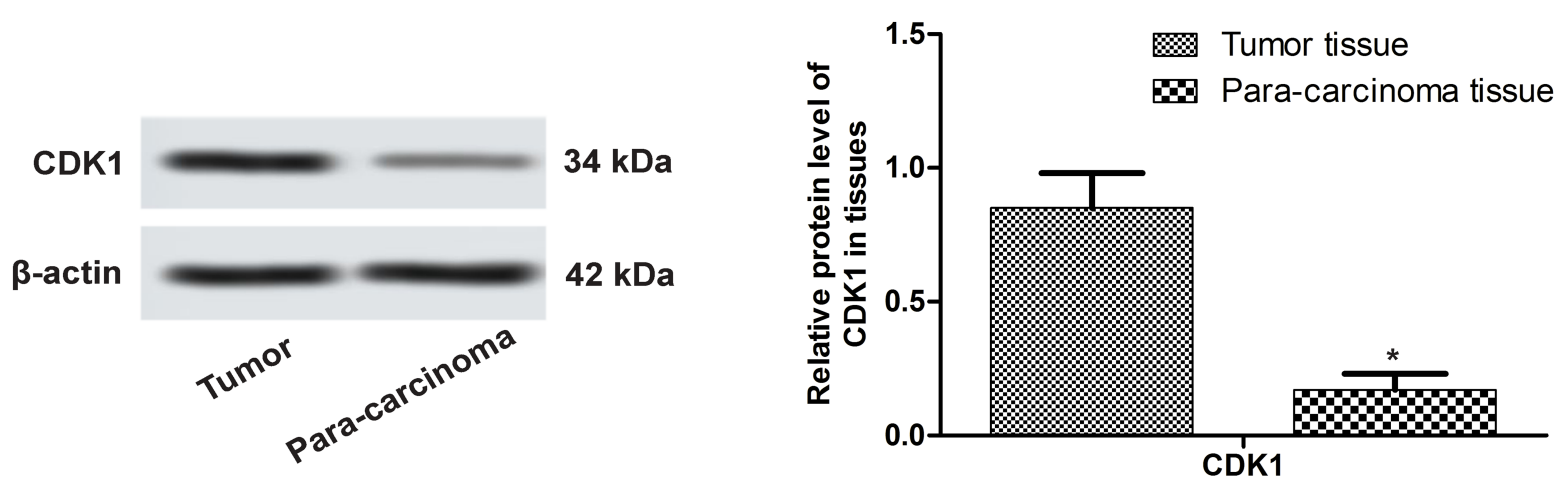

Figure I miR-96-5p low-expression and CDKI high-expression in NPC tissues and cells. (A) The mRNA levels of miR-96-5p or CDKI in NPC tissues and para-carcinoma normal tissues were examined by RT-qPCR. (B) The mRNA levels of miR-96-5p or CDKI in NP69 cells and NPC cells (CNE-2Z, HNE-2, C666-I, 5-8F and SUNE-I) were examined by RT-qPCR. (C) The protein level of CDKI was detected by Western blot. Data are presented as mean \pm SD. $* P<0.05$, **P $<0.0$ I. GAPDH or U6 as the internal control. All operations were performed in triplicate.

more obvious. After co-transfection mimic and pc-CDK1, miR-96-5p inhibited the decrease of cell $\mathrm{SF}$, compared with CDK1 group (Figure 5B). In addition, CNE-2Z-DDP cells were exposed to different doses of DDP $(0,5,10,15$ or $20 \mu \mathrm{M})$. The results of Figure 5D demonstrated that miR-96-5p 
A

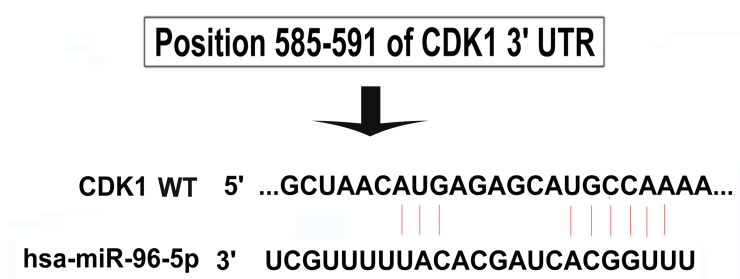

C

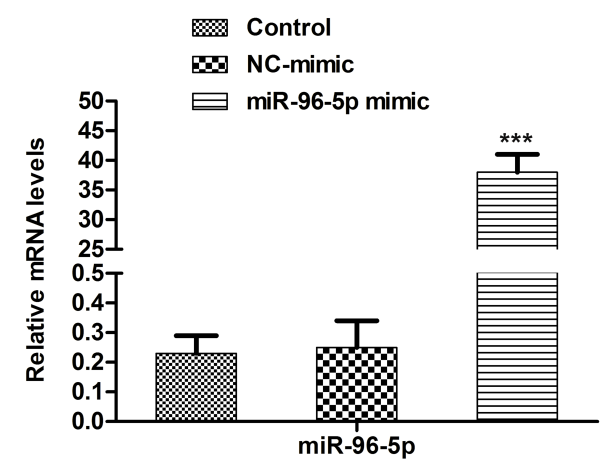

B

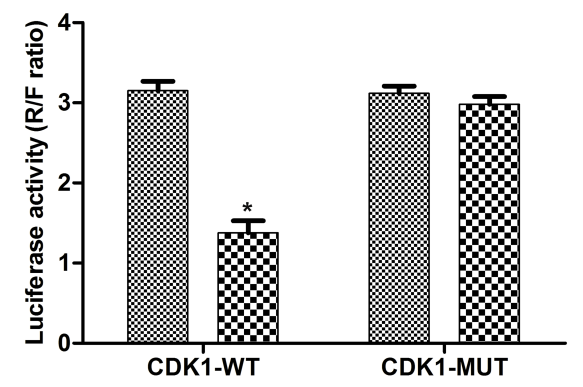

NC-mimic

$\mathbf{m i R - 9 6 - 5 p ~ m i m i c ~}$

Figure 2 miR-96-5p directly targets CDKI. (A) The predicted binding sites between miR-96-5p and CDKI 3' UTR employing TargetScan software. (B) Luciferase activity of WT or MUT-CDKI was detected using luciferase reporter. (C) CNE-2Z cells were transfected with miR-96-5p mimic or NC, the mRNA level of miR-96-5P was detected by RT-qPCR assay. (D) CNE-2Z cells were transfected with miR-96-5p mimic/NC, pc-CDKI or co-transfection, the mRNA level of CDKI was detected by RT-qPCR assay. Data are presented as mean $\pm \mathrm{SD}$. ${ }^{*} \mathrm{P}<0.05$, ${ }^{*} \mathrm{P}<0.0 \mathrm{I}$, ${ }^{* * *} \mathrm{P}<0.00 \mathrm{I}$ vs control; ${ }^{*} \mathrm{P}<0.05 \mathrm{vs} \mathrm{Pc}-\mathrm{CDKI}$. U6 as the internal control. All operations were performed in triplicate.

A

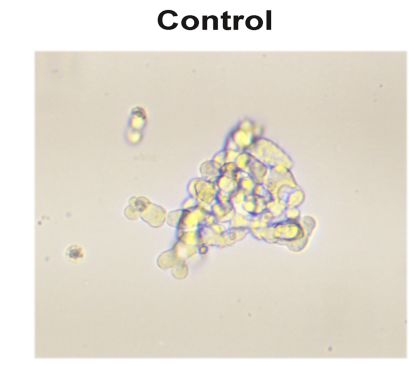

B

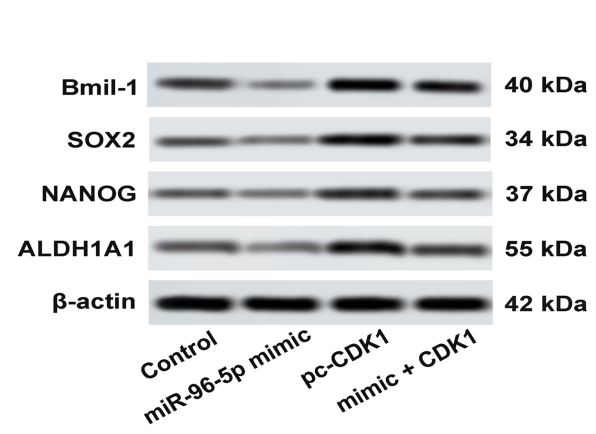

miR-96-5p mimic

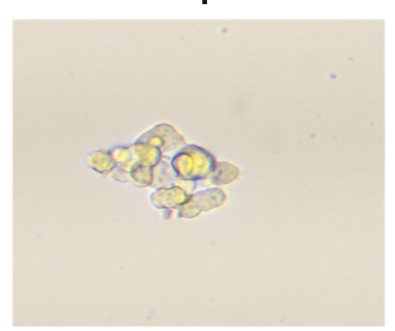

C

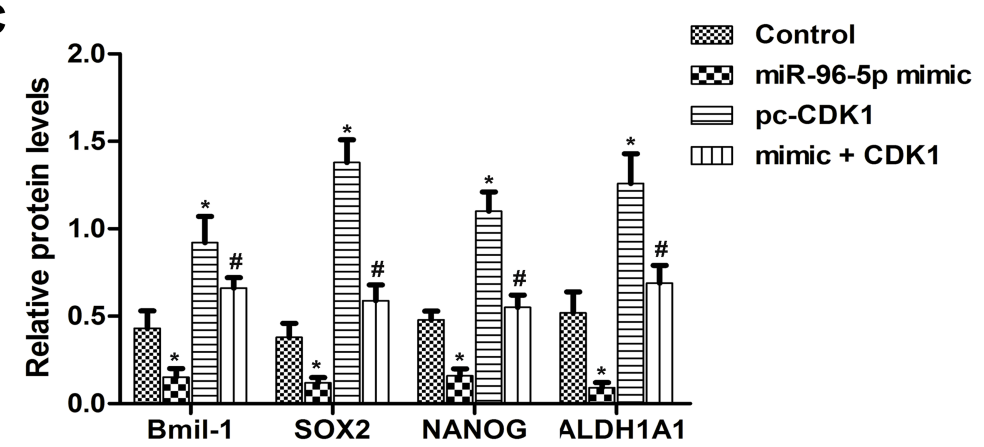

Figure 3 Effect of miR-96-5p on cell stem-like property. (A) sphere formation of CNE-2Z cells in different groups (200x). (B, C) The protein levels of Bmi-I, SOX2, NANOG and ALDHIAI were detected by Western blot. Data are presented as mean \pm SD. ${ }^{*} \mathrm{P}<0.05$ vs control; ${ }^{*} \mathrm{P}<0.05$ vs $p c-C D K I$. $\beta$-actin as the internal control. All operations were performed in triplicate.

overexpression promoted the clonal formation ability of CNE-2Z-DDP cells, compared with the NC. Also, CCK-8 showed that CDK1 overexpression restrained
CNE-2Z-DDP cells' viability with gradient doses of DDP, but this effect was reversed by miR-96-5p mimic (Figure 5C). 
A
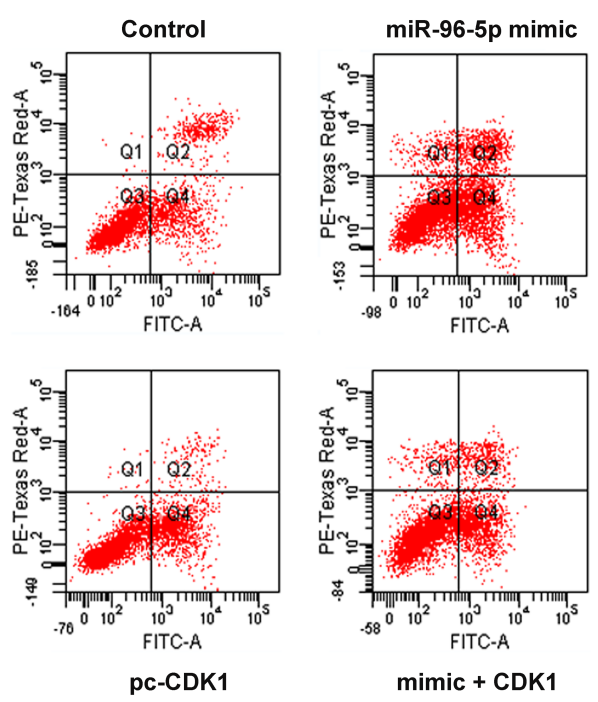

B

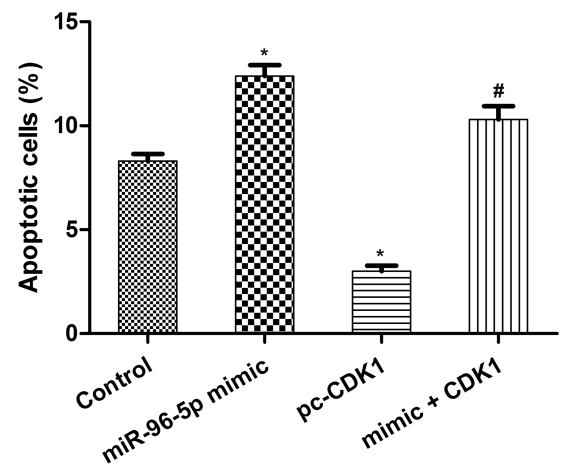

\begin{abstract}
\%ontrol
MiR-96-5p mimic

戸 $\mathrm{pc}-\mathrm{CDK} 1$
\end{abstract}

سा mimic + CDK1

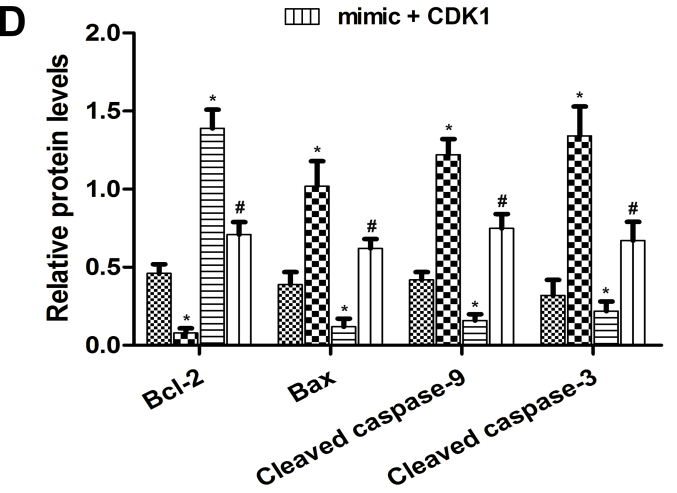

E
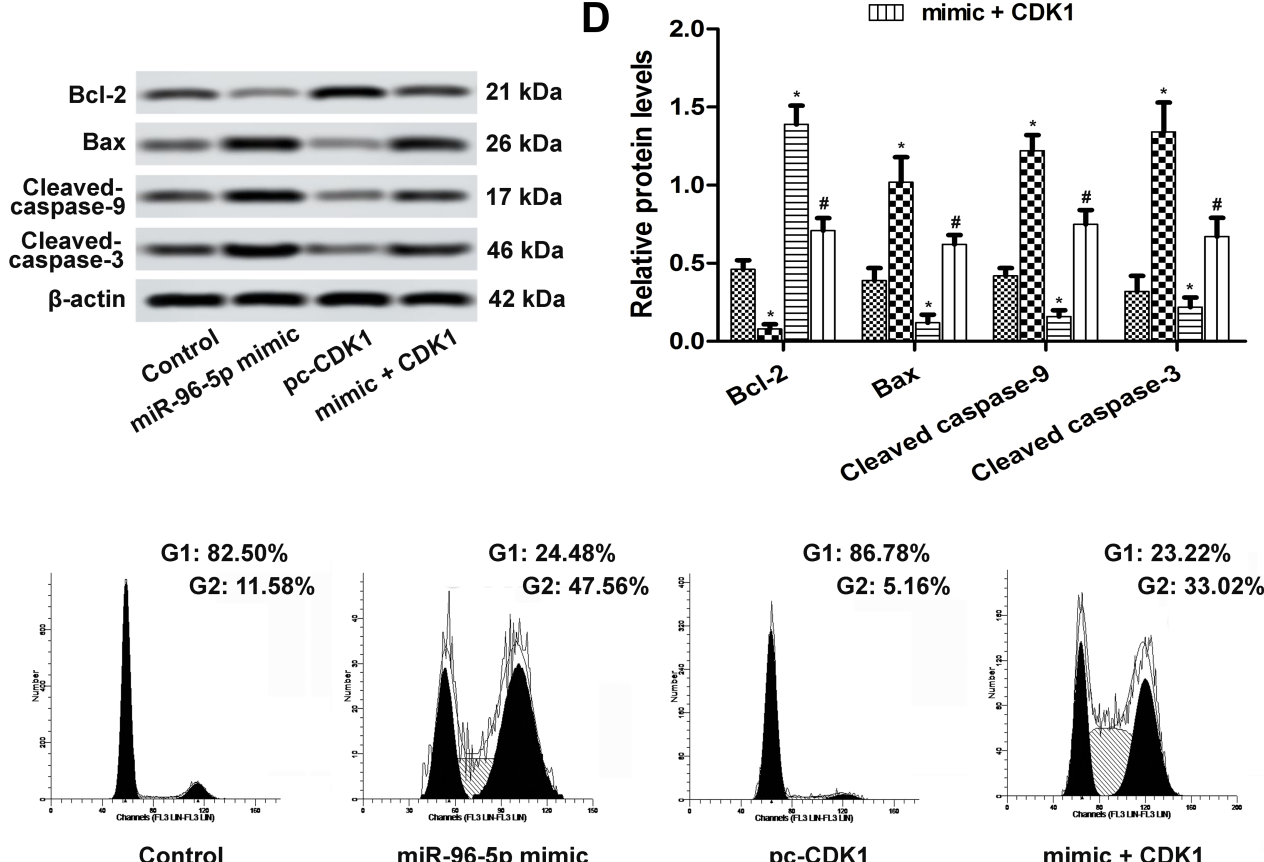

$\mathbf{F}$

G
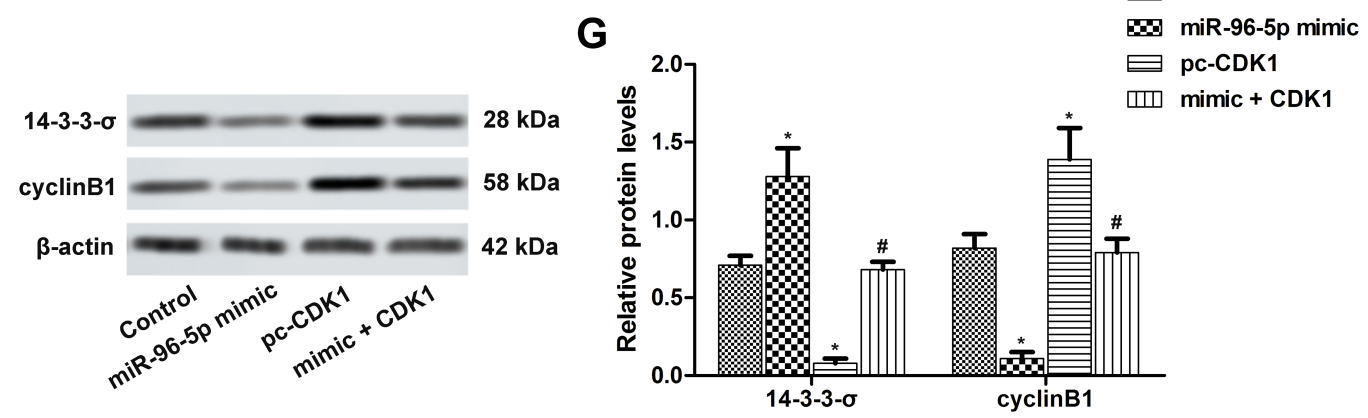

Figure 4 Effect of miR-96-5p on cell apoptosis and cell cycle. (A, B) Apoptosis of CNE-2Z cells was detected by flow cytometry. (C, D) The protein levels of Bcl-2, Bax, cleaved-Caspase-3 and cleaved-Caspase-9 were detected by Western blot. (E) Cells cycle of CNE-2Z cells was analyzed by flow cytometry. (F, G) The protein levels of I43-3- $\sigma$ and cyclinBI were detected by Western blot. Data are presented as mean \pm SD. ${ }^{*} \mathrm{P}<0.05$ vs control; ${ }^{*} \mathrm{P}<0.05$ vs $\mathrm{pc}-\mathrm{CDKI}$. $\beta$-actin as the internal control. All operations were performed in triplicate. 
A

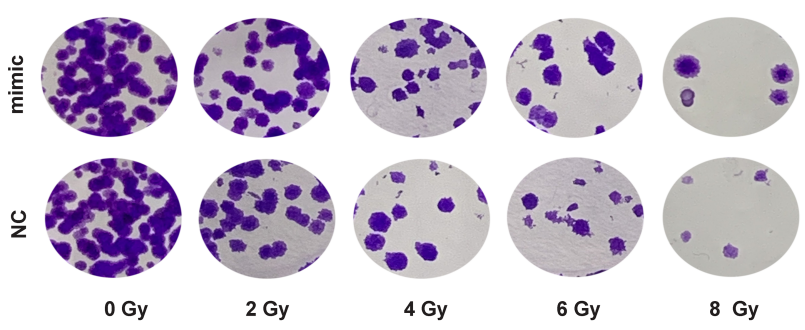

C

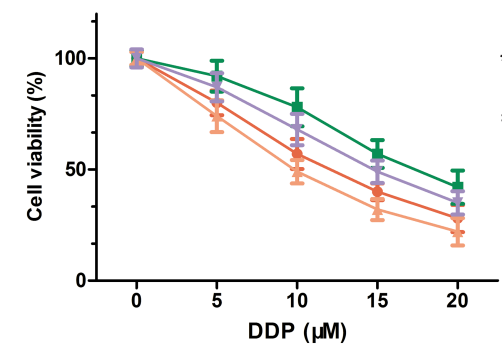

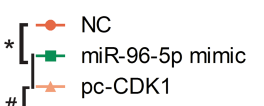

$\#\left[\begin{array}{cc}-1 \\ \rightarrow-\operatorname{mimic}+\mathrm{CDK} 1\end{array}\right.$
B

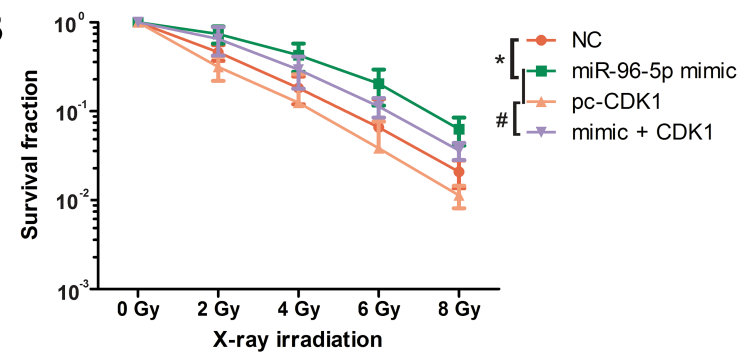

D

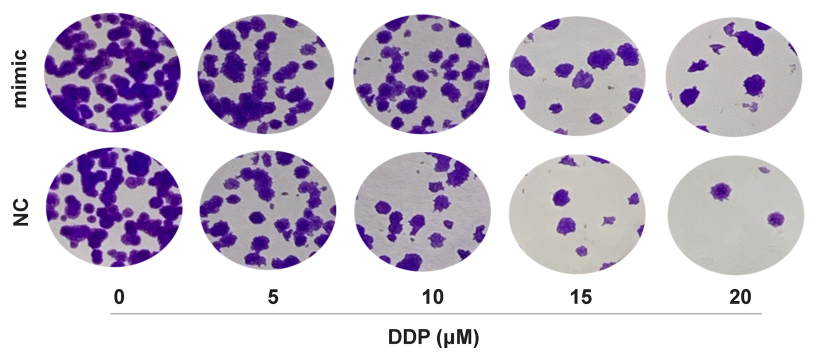

Figure 5 Effect of miR-96-5p on the chemoradiotherapy sensitivity of CNE-2Z cells. (A) Colony formation ability of CNE-2Z-R cells were detected with irradiation gradient doses (0, 2, 4, 6 or 8 Gy). (B) Survival curve of CNE-2Z-R cells irradiated at gradient doses (0, 2, 4, 6 or 8 Gy). (C) Cell viability was determine by CCK-8 assay with DDP gradient doses $(0,5,10,15$ or $20 \mu \mathrm{M})$. (D) Colony formation ability of CNE-2Z-DDP cells exposed at DDP gradient doses (0, 5, 10,15 or $20 \mu \mathrm{M})$. Data are presented as mean $\pm \mathrm{SD}$. $* \mathrm{P}<0.05$ vs NC; ${ }^{\#} \mathrm{P}<0.05$ vs $\mathrm{pc}-\mathrm{CDKI}$. All operations were performed in triplicate.

\section{miR-96-5p Overexpression Suppressed Tumor Formation in vivo}

To examine the anti-tumor effect of miR-96-5p in vivo, CNE-2Z cells transfected with miR-96-5p mimics were used to establish a xenograft tumor model. As shown in Figure 6A-C, we observed that miR-96-5p mimic decreased the tumor weight and size, compared with NC.
In addition, the mRNA level of miR-96-5p was elevated while CDK1 was reduced in tumor tissues (Figure 6D). Compared with NC, Western blot analysis showed that elevated miR-96-5p significantly inhibited the expression of NANOG, ALDH1A1 and cyclinB1 proteins in tumor tissues, but increased the expression of p53 and cleaved Caspase-3 (Figure 6E). Furthermore, the data from

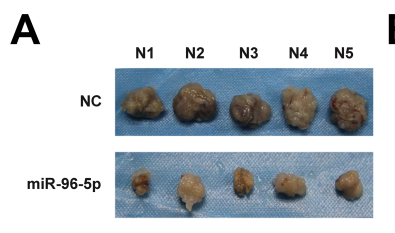

$\mathbf{E}$

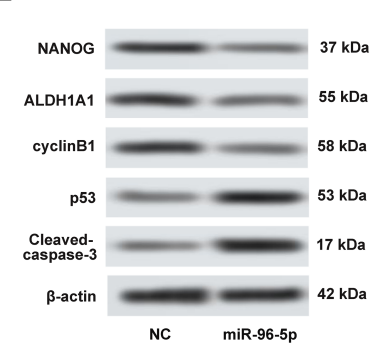

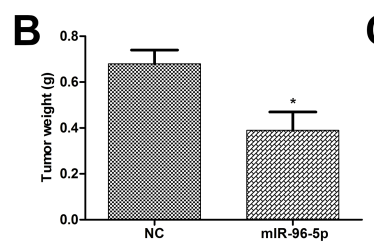

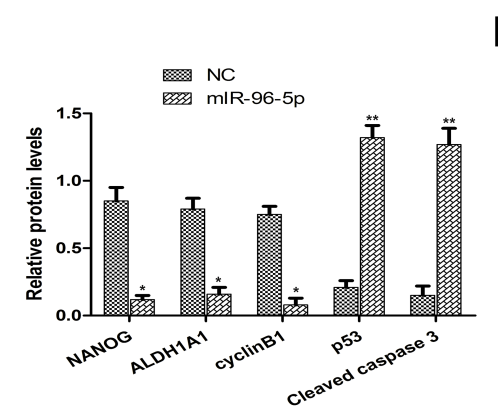

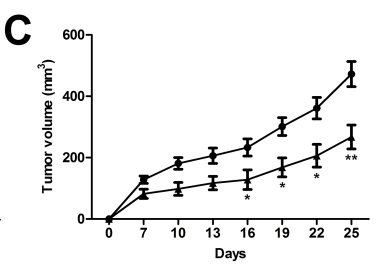

$\mathbf{F}$

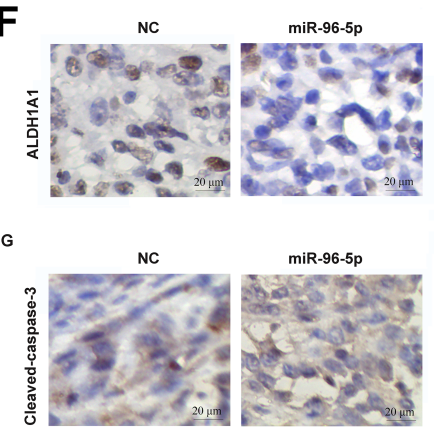

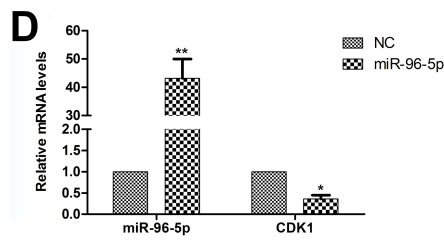
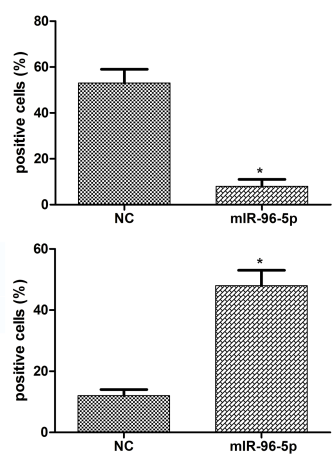

Figure 6 miR-96-5p alleviated tumor growth in vivo. (C) Tumor weights and volumes were measured. (D) The mRNA levels of miR-96-5p or CDKI in xenograft tumor tissues were examined by RT-qPCR. (E) The protein levels of NANOG, ALDHIAI, cyclinBI, p53 and cleaved-Caspase-3 were detected by Western blot. (F, G) The positive expression of ALDHIA and cleaved-Caspase-3 were detected by IHC (200x). Data are presented as mean \pm SD. $* \mathrm{P}<0.05$, **P $<0.0 \mathrm{I}$ vs NC. GAPDH, U6 or $\beta$-actin as the internal control. All operations were performed in triplicate. 
Figure $6 \mathrm{~F}$ and $\mathrm{G}$ showed that miR-96-5p overexpression significantly increased the positive cell number of cleaved Caspase- 3 in tumor tissues and decreased the number of ALDH1A1 cells, compared with NC.

\section{Discussion}

Nasopharyngeal carcinoma is a malignant tumor originating from the nasopharyngeal epithelium, which is prone to local recurrence and distant metastasis. Although radiotherapy or induction chemotherapy is the basic method of clinical treatment of NPC, ${ }^{4}$ it is necessary to find new tumor markers and targets for the treatment of advanced $\mathrm{NPC} .^{20}$ In this study, we found that miR-96-5p was lowly expressed in NPC. Moreover, the abnormal expression of miR-96-5p was related to the stem-like properties, apoptosis, cell cycle arrest and radiochemotherapy sensitivity of NPC cells. Animal experiments showed that miR-96-5p overexpression inhibited tumor formation in tumorbearing mice. Notably, we also found that CDK1 was the miR-96-5p target, and miR-96-5p regulated the progression of NPC by targeting CDK1.

Previous studies have confirmed that abnormal expression of miRNAs is closely related to tumorigenesis and development, such as apoptosis, cell cycle, cell proliferation, metastasis and radiochemotherapy resistance. ${ }^{6,7,21}$ It was observed that miR-96-5p expression was dysregulated in some cancers. ${ }^{8-10}$ Here, we detected that the relative mRNA level of miR-96-5p was remarkably downregulated in NPC tissues and cells. miR-96-5p overexpression could significantly promoted the apoptosis and cell cycle arrest of NPC cells. Additionally, luciferase activity was decreased in the CDK1 3' UTR (wt) by miR-96-5p mimic. CDK1 is highly conserved in eukaryotic cell cycle regulation. ${ }^{22}$ The G2/M transition is mainly regulated by the CDK1/Cyclin B1 complex, which is blocked by the depletion of CDK1. ${ }^{11,12}$ Notably, we found that the positive effects of miR-96-5p induced-apoptosis and cell cycle arrest of NPC cells could be reversed by CDK1 overexpression. Thus, miR-96-5p modulated NPC cell cycle arrest and apoptosis through suppressing the expression of CDK1.

Cancer Stem Cells (CSCs) are a group of tumorinitiating cells that can self-renew and mediate tumorigenesis and metastasis. ${ }^{23}$ Initially, CSCs were found to have the ability to self-renew and form new tumors in acute myeloid leukemia. ${ }^{24-26}$ Later, the study of CSCsextended to solid tumors, such as brain cancer, ${ }^{27}$ lung cancer $^{28}$ and colon cancer. ${ }^{29}$ Unlike tumor cells, CSCs differentiate slowly on their own but are resistant to radiotherapy and chemotherapy. Conventional chemotherapy drugs only kill ordinary tumor cells, not CSCs. Therefore, CSCs are considered to be the root cause of NPC recurrence. Su et al identified $\mathrm{CD} 44^{+}$-like cells in human NPC cell lines. ${ }^{30,31}$ In our study, the microsphere formation culture obtained the stem-like characteristics of NPC cells. Sox 2 is a critical member of the Sox family involved in regulating the transformation of pluripotent stem cells. ${ }^{32}$ NANOG is another critical stem cell transcription factor, together with Sox2, maintains the pluripotent differentiation potential of human embryonic stem cells as well as cell differentiation in early embryonic development. Our results found that overexpression of miR-96-5p significantly reduced the pellet size of stem-like cells and inhibited the expression of stem-like marker proteins (Bmi-1, SOX2, NANOG and ALDH1A1), suggesting that miR-96-5p overexpression inhibited NPC stem cells to form large tumor spheres. ${ }^{33}$

Actually, miRNAs can modify cellular responses to irradiated or precise drugs through mechanisms such as interference DNA repair and targets. ${ }^{34,35}$ Interestingly, studies reported that up-regulated miRNA-21 promoted resistance of NPC cells to cisplatin-induced apoptosis. ${ }^{20} \mathrm{CDK} 1$ mediates cell cycle arrest in response to DNA damage in response to radiation. ${ }^{36}$ In this study, we found that up-regulation of miR-96-5p increased the radiochemical sensitivities of NPC cells by targeting CDK1, overexpression CDK1 might be correlated with radiochemical sensitivities. ${ }^{37}$

\section{Conclusion}

Collectively, the present study demonstrated that miR-96$5 \mathrm{p}$ was down-regulated and CDK1 up-regulated in NPC. CDK1 was identified as a direct target of miR-96-5p. Our data highlighted the tumor inhibition of miR-96-5p in NPC by targeting CDK1, it provided new biochemical markers for clinical examination or treatment of NPC.

\section{Funding}

This work was supported by the Research Projects Fund of Southwest Medical University (No. 2018-ZRQN-106 and 2018-ZRQN-168), and the Project Fund of Hospital (T.C. M) Affiliated to Southwest Medical University (No. 2018XYLH-045 and 2018-XYLH-048).

\section{Disclosure}

The authors report no conflicts of interest in this work. 


\section{References}

1. He R, Zheng H, Wang Q, et al. The role of long non-coding RNAs in nasopharyngeal carcinoma: as systemic review. Oncotarget. 2017;8:16075-16083. doi:10.18632/oncotarget.14211

2. Chen W, Zheng R, Baade PD, et al. Cancer statistics in China, 2015. CA Cancer J Clin. 2016;66(2):115-132. doi:10.3322/caac.21338

3. Fang $\mathrm{Y}$, Zhu $\mathrm{X}$, Wang J, et al. MiR-744 functions as a proto-oncogene in nasopharyngeal carcinoma progression and metastasis via transcriptional control of ARHGAP5. Oncotarget. 2015;6 (15):13164-13175. doi:10.18632/oncotarget.3754

4. Chen YP, Chan ATC, Le QT, Blanchard P, Sun Y, Ma J. Nasopharyngeal carcinoma. Lancet. 2019;394(10192):64-80. doi:10.1016/S0140-6736(19)30956-0

5. He J, Ye W, Kou N, et al. MicroRNA-29b-3p suppresses oral squamous cell carcinoma cell migration and invasion via IL32/AKT signalling pathway. $J$ Cell Mol Med. 2020;24(1):841-849. doi:10.1111/jcmm.14794

6. Wang B, Li W, Liu H, et al. miR-29b suppresses tumor growth and metastasis in colorectal cancer via downregulating Tiam1 expression and inhibiting epithelial-mesenchymal transition. Cell Death Dis. 2014;5(7):e1335. doi:10.1038/cddis.2014.304

7. Wu S, Zhang G, Li P, et al. miR-198 targets SHMT1 to inhibit cell proliferation and enhance cell apoptosis in lung adenocarcinoma. Tumour Biol. 2016;37(4):5193-5202. doi:10.1007/s13277-0154369-z

8. Liu B, Zhang J, Yang D. miR-96-5p promotes the proliferation and migration of ovarian cancer cells by suppressing Caveolae1. J Ovarian Res. 2019;12:57.

9. Santhi WS, Prathibha R, Charles S, et al. Oncogenic microRNAs as biomarkers of oral tumorigenesis and minimal residual disease. Oral Oncol. 2013;49:567-575. doi:10.1016/j.oraloncology.2013.01.001

10. He PY, Yip WK, Jabar MF, Mohtarrudin N, Dusa NM, Seow HF. Effect of the miR-96-5p inhibitor and mimic on the migration and invasion of the SW480-7 colorectal cancer cell line. Oncol Lett. 2019;18(2):1949-1960. doi:10.3892/ol.2019.10492

11. Hu X, Moscinski LC. Cdc2: a monopotent or pluripotent CDK? Cell Prolif. 2011;44:205-211. doi:10.1111/j.1365-2184.2011.00753.x

12. Enserink JM, Kolodner RD. An overview of Cdk1-controlled targets and processes. Cell Div. 2010;5:11. doi:10.1186/1747-1028-5-11

13. Allan LA, Clarke PR. Phosphorylation of caspase-9 by CDK1/cyclin B1 protects mitotic cells against apoptosis. Mol Cell. 2007;26:301-310. doi:10.1016/j.molcel.2007.03.019

14. Matthess Y, Raab M, Sanhaji M, Lavrik IN, Strebhardt K. Cdk1/ cyclin B1 controls Fas-mediated apoptosis by regulating caspase-8 activity. Mol Cell Biol. 2010;30(24):5726-5740. doi:10.1128/ MCB.00731-10

15. Neganova I, Tilgner K, Buskin A, et al. CDK1 plays an important role in the maintenance of pluripotency and genomic stability in human pluripotent stem cells. Cell Death Dis. 2014;5(11):e1508. doi:10.1038/cddis.2014.464

16. Otto T, Sicinski P. Cell cycle proteins as promising targets in cancer therapy. Nat Rev Cancer. 2017;17(2):93-115. doi:10.1038/nrc.20 16.138

17. Li S, Zhang XM, Zhang RK, et al. Hippo pathway contributes to cisplatin resistant-induced EMT in nasopharyngeal carcinoma cells. Cell Cycle. 2017;16(17):1601-1610. doi:10.1080/15384101.2017. 1356508

18. Yu ZW, Wang GB, Zhang CL, et al. LncRNA SBF2-AS1 affects the radiosensitivity of non-small cell lung cancer via modulating microRNA-302a/MBNL3 axis. Cell Cycle. 2020;19(3):300-316. doi:10.1080/15384101.2019.1708016

19. Shah M, Cardenas R, Wang B, et al. HOXC8 regulates self-renewal, differentiation and transformation of breast cancer stem cells. Mol Cancer. 2017;16(1):38. doi:10.1186/s12943-017-0605-z
20. Zhong Q, Liu ZH, Lin ZR, et al. The RARS-MAD1L1 fusion gene induces cancer stem cell-like properties and therapeutic resistance in nasopharyngeal carcinoma. Clin Cancer Res. 2018;24:659-673. doi:10.1158/1078-0432.CCR-17-0352

21. Banno K, Iida M, Yanokura M, Kisu I, Iwata T, Tominaga E, Tanaka K, Aoki D. MicroRNA in cervical cancer: oncomiRs and tumor suppressor miRs in diagnosis and treatment. Sci World J. 2014;2014:178075. doi:10.1155/2014/178075

22. Deota S, Rathnachalam S, Namrata K, et al. Allosteric regulation of cyclin-B binding by the charge state of catalytic lysine in CDK1 is essential for cell-cycle progression. $J$ Mol Biol. 2019;431 (11):2127-2142. doi:10.1016/j.jmb.2019.04.005

23. Adorno-Cruz V, Kibria G, Liu X, et al. Cancer stem cells: targeting the roots of cancer, seeds of metastasis, and sources of therapy resistance. Cancer Res. 2015;75:924-929. doi:10.1158/0008-5472. CAN-14-3225

24. Lapidot T, Sirard C, Vormoor J, et al. A cell initiating human acute myeloid leukaemia after transplantation into SCID mice. Nature. 1994;367(6464):645-648. doi:10.1038/367645a0

25. Mehrotra B, George TI, Kavanau K, et al. Cytogenetically aberrant cells in the stem cell compartment (CD34+lin-) in acute myeloid leukemia. Blood. 1995;86:1139-1147.

26. Haase D, Feuring-Buske M, Konemann S, et al. Evidence for malignant transformation in acute myeloid leukemia at the level of early hematopoietic stem cells by cytogenetic analysis of CD34+ subpopulations. Blood. 1995;86:2906-2912. doi:10.1182/blood. V86.8.2906.2906

27. Singh SK, Clarke ID, Terasaki M, et al. Identification of a cancer stem cell in human brain tumors. Cancer Res. 2003;63:5821-5828.

28. Eramo A, Lotti F, Sette G, et al. Identification and expansion of the tumorigenic lung cancer stem cell population. Cell Death Differ. 2008;15(3):504-514. doi:10.1038/sj.cdd.4402283

29. Todaro M, Alea MP, Di Stefano AB, et al. Colon cancer stem cells dictate tumor growth and resist cell death by production of interleukin-4. Cell Stem Cell. 2007;1(4):389-402. doi:10.1016/j. stem.2007.08.001

30. Su J, Xu XH, Huang Q, et al. Identification of cancer stem-like CD44 + cells in human nasopharyngeal carcinoma cell line. Arch Med Res. 2011;42(1):15-21. doi:10.1016/j.arcmed.2011.01.007

31. Janisiewicz AM, Shin JH, Murillo-Sauca O, et al. CD44+ cells have cancer stem cell-like properties in nasopharyngeal carcinoma. Int Forum Allergy Rhinol. 2012;2(6):465-470. doi:10.1002/alr.21068

32. Castel P, Ellis H, Bago R, et al. PDK1-SGK1 signaling sustains AKTindependent mTORC1 activation and confers resistance to PI3K $\alpha$ inhibition. Cancer Cell. 2016;30(2):229-242. doi:10.1016/j.ccell.2016.06.004

33. Cheung CC, Lun SW, Chung GT, et al. MicroRNA-183 suppresses cancer stem-like cell properties in EBV-associated nasopharyngeal carcinoma. BMC Cancer. 2016;16:495. doi:10.1186/s12885-016-2525-5

34. Zhao H, Liu S, Wang G, et al. Expression of miR-136 is associated with the primary cisplatin resistance of human epithelial ovarian cancer. Oncol Rep. 2015;33(2):591-598. doi:10.3892/or.2014.3640

35. Chen H, Yao XJ, Di XK, et al. MiR-450a-5p inhibits autophagy and enhances radiosensitivity by targeting dual-specificity phosphatase 10 in esophageal squamous cell carcinoma. Cancer Lett. 2020;483:114-126. doi:10.1016/j.canlet.2020.01.037

36. Alexandrou AT, Li JJ. Cell cycle regulators guide mitochondrial activity in radiation-induced adaptive response. Antioxid Redox Signal. 2014;20;20(9):1463-1480. doi:10.1089/ars.2013.5684

37. Liu HM, Tan HY, Lin Y, Xu BN, Zhao WH, Xie YA. MicroRNA1271-5p inhibits cell proliferation and enhances radiosensitivity by targeting CDK1 in hepatocellular carcinoma. J Biochem. 2020;167 (5):513-524. doi:10.1093/jb/mvz114 


\section{Publish your work in this journal}

OncoTargets and Therapy is an international, peer-reviewed, open access journal focusing on the pathological basis of all cancers, potential targets for therapy and treatment protocols employed to improve the management of cancer patients. The journal also focuses on the impact of management programs and new therapeutic

Submit your manuscript here: https://www.dovepress.com/oncotargets-and-therapy-journa agents and protocols on patient perspectives such as quality of life, adherence and satisfaction. The manuscript management system is completely online and includes a very quick and fair peer-review system, which is all easy to use. Visit http://www.dovepress.com/ testimonials.php to read real quotes from published authors. 